\title{
DFT-D Investigation of the Interaction Between Ir(III) Based Photosensitizers and Small Silver Clusters $\mathrm{Ag}_{n}$$$
(n=2-20,92)
$$

\author{
Olga S. Bokareva ${ }^{\mathrm{a}, *}$, Oliver Kühn ${ }^{\mathrm{a}}$ \\ ${ }^{a}$ Institut für Physik, Universität Rostock, D-18051 Rostock, Germany
}

\begin{abstract}
A dispersion-corrected density functional theory study of the photosensitizer $\left[\operatorname{Ir}(\text { ppy })_{2}(\mathrm{bpy})\right]^{+}$and its derivatives bound to silver clusters $\operatorname{Ag}_{n}(n=2-20$, 92 ) is performed. The goal is to provide a new system-specific set of $C_{6}$ interaction parameters for $\mathrm{Ag}$ and Ir atoms. To this end a QM:QM scheme is employed using the PBE functional and RPA as well as MP2 calculations as reference. The obtained $C_{6}$ coefficients were applied to determine dissociation curves of selected IrPS $-\mathrm{Ag}_{n}$ complexes and binding energies of derivatives containing oxygen and sulphur as heteroatoms in the ligands. Comparing different $C_{6}$ parameters it is concluded that RPA-based dispersion correction produces binding energies close to standard D2 and D3 models, whereas MP2-derived parameters overestimate these energies.
\end{abstract}

Keywords: dispersion interaction, density functional theory, organic/inorganic hybrid systems, binding energies

\section{Introduction}

Inorganic/organic hybrid systems comprised of small metal nanoparticles and different organic adsorbates like peptides and dyes represent a fascinating topic with prospective applications in, e.g., catalysis and bioelectronics (for reviews, see Refs. [1, 2, 3, 4]).

Despite considerable progress, electronic structure calculations of such hybrid systems with non-covalent interactions still pose a challenge for quantum

*Email: obokareva@gmail.com 
chemical methods that are known for their moderate computational costs. In particular, standard density functional theory (DFT) techniques fail to predict the adsorption energies for aromatic molecules on noble metal surfaces in agreement with experiments [5, 6, 7]. Often this discrepancy can be attributed to the inadequate treatment of the dispersion interaction within most currently available functionals [8, 9, 10, 11, 12]. This holds true in particular for weakly physisorbed adsorbates, where the incorrect estimation of dispersion interaction might have a much more pronounced impact on the quality of results than in case of covalently bound complexes [5, 6, 7].

Since the dispersion interaction is the main attractive force holding weakly bound molecules together, it stands in modern literature for the more general van der Waals (vdW) interaction, which includes different types of intermolecular interactions such as electrostatic (permanent multipole-permanent multipole), induction (permanent multipole-induced multipole), and dispersion (induced multipole-induced multipole). Strictly speaking, only manyelectron quantum chemistry methods can reliably describe the dispersion term, as it arises from the correlated motion of electrons. Hence, for computational reasons, the direct calculation of $\mathrm{vdW}$ interactions is limited to small systems. That is why alternative approximate approaches to the investigation of weakly-bounded systems at reasonable computational costs are warrant and the extension of standard DFT to account for dispersion effects is an active area of research (for a review, see Refs. [13, 12, 14]). Here, the strategies can be classified according to the basis on which the dispersion term is founded, i.e. the effective one electron potential, the interaction between pairs of atoms with $C_{6}$ atomic coefficients or the inclusion of non-local terms in the exchange-correlation (XC) kernel.

The first approach includes dispersion-corrected atom-centered potentials (DCACP) [15, 16] as well as its local variants LAP [17] and CAP [18] and the recently proposed empirical force correcting atom-centred potentials (FCACP's) [19]. It makes use of tuned effective core potentials placed at each atom in the way that the dispersion energy is represented by a sum of one-electron terms.

The idea of representing the dispersion interaction by pairwise atomic $-C_{n} R^{-n}$ potentials with proper damping functions was explored by many authors. In the exchange-hole dipole moment (XDM) approach [20, 21, 22, 23, 24, 25, 26], the instantaneous dipole formed between an electron and its exchange hole is used to express the dispersion interaction between nonoverlapping charge densities using the Casimir-Polder relation [27]. In most 
of the recent implementations, the pairwise potentials includes terms up to tenth order $\left(C_{6}, C_{8}\right.$ and $C_{10}$ coefficients). The impact of the chemical environment on the dispersion coefficients is taken into account through Hirshfeld partitioning.

In the DFT-D2 approach of Grimme, $C_{6}$ parameters for the first part of the periodic table are derived from calculations of ionization potentials and static dipole polarizabilities for single atoms and proved to give an adequate description of non-covalent interactions. For heavy atoms, however, no reliable parameters within the D2 model are available [28]. As proposed by Grimme, unknown coefficients could be deduced from atomic properties by the London formula [29]. An alternative way to obtain dispersion corrections is the so-called hybrid QM:QM approach [30, 31, 32]. In this method, the dispersion energy is considered as the difference between the adsorption energies for adsorbate-substrate complex obtained with ab initio theory and DFT. For absorption of pyridine on gold clusters, it has been shown by Tonigold and Groß that QM:QM employing a MP2 (second order Møller-Plesset perturbation theory) reference gave substantially better agreement with experiment compared to standard D2 [33].

Later, Grimme has introduced the much advanced DFT-D3 method, taking into account the surrounding of the atoms by means of coordination numbers in contrast to DFT-D2 where by construction the dispersion coefficients are not system-dependent. In this approach, $C_{6}$ coefficients were derived from averaged dipole polarizabilities at imaginary frequencies calculated with time-dependent DFT (TD-DFT), while eighth order coefficients $C_{8}$ follow from a simple recursion rules for the higher-multipole terms [34]. DFT-D3 has been shown to provide higher accuracy and broader applicability for 94 elements of the periodic table than the earlier versions.

Tkatchenko and Scheffler developed a method (TS or DFT-vdW) 35] of calculating dispersion coefficients and $\mathrm{vdW}$ radii from the ground-state molecular or condensed matter electron density. Here, the starting point is a high-level ground-state calculation of free-atom properties. In a second step the effect of the surroundings is taken into account by considering Hirshfeld volumes and the electron density for the whole system. Further, the self-consistent screening is accounted for to reproduce the anisotropy of the molecular static polarizability [36]. In the DFT-vdWsurf variant the manybody collective response is analyzed for cases of adsorbates on a surface [37]. In that respect, the DFT $+\mathrm{vdW}$ group is more akin to XDM, because it gives $C_{6}$ coefficients that are dependent on the local environment, but not only on 
coordination number of each atom like in DFT-D3.

In passing we note that in order to avoid double counting effects at medium and short distances a linkage between the long-range dispersion term and the short-range DFT energy must be introduced. In all DFT+dispersion variants this is achieved by multiplying the dispersion energy by a damping function that provides a smooth cut-off at short distances. In general, this type of dispersion correction can by applied to different DFT variants, but corresponding empirical coefficients should be properly calibrated.

Finally, a more rigorous development (noted as vdW-DFs) sets the focus on the determination of an explicit non-local correlation functional from first principles. Currently, the following versions are used vdW-DF [38, 39, 40], VV09, and VV10 [41, 42]. The exchange-correlation energy includes a nonlocal term, which is undamped (in modern versions) and contributes at short distances as well. The kernel used to compute the non-local term is typically based on a local approximation to the dipole polarizability at imaginary frequencies. This results in a long-range part of the dispersion energy through the Casimir-Polder relation [27], similar to modern DFT-D approaches to $C_{6}$ coefficients. The similarity between vdW-DF and DFT-D can also be seen from the fact, that the non-local term of the former approach is typically computed non-self-consistently, resulting in some additional contribution to the DFT energy. Both routes describe the long-range vdW interaction and, at atomic overlap regions, link it to a standard exchange-correlation functional. The advantage of vdW-DF is that dispersion effects are calculated based on the charge density, that is, in cases of charge transfer the effect of dispersion is naturally included. Recently, some progress in increasing their efficiency has been achieved making the computational costs comparable to those of standard DFT-GGA calculations what would enable their future application to large systems [43, 44, 45, 42]. For example, vdW-inclusive DFT methods allow to reliably model adsorption of molecules on surfaces [46], for review see [13].

Although benchmarks for validation of new dispersion-corrected DFT approaches have been reported (see, e.g., Refs. [25, 47, 48, 49, 50, 51] and references therein), mainly non-metal containing systems have been considered such as the S22 standard set and nucleobase pairs. Exceptions include the application of DFT-D to the adsorption of aliphatic and aromatic molecules on metal surfaces like gold, silver, palladium, and copper [33, 34, 52, 53, 54, 55, 56, 57, 58], which gave a good agreement with experimental adsorption energies, in contrast to conventional DFT. However, 
note that for $\mathrm{AuL}_{x}-\mathrm{C}_{n} \mathrm{H}_{m}$ model systems, for instance, the DFT-D3 performance is comparable with that of conventional DFT [59]. An alternative for improving the DFT-D performance is to use system-dependent $C_{6}$ coefficients, rescaled on the basis of an embedding model; examples include the adsorption of small organic molecules on $\mathrm{MgO}$ and $\mathrm{NaCl}$ surfaces [60]. In conclusion, the usage of dispersion-corrected DFT approaches for systems including metal-containing surfaces or especially clusters clearly needs further investigation and comprehensive benchmarking in order to become a standard method comprising the modest computational costs of DFT with accurate predictions.

The present study aims at establishing an empirical dispersion correction for the system $\left[\operatorname{Ir}(\text { ppy })_{2}(\text { bpy })\right]^{+}(\operatorname{IrPS})$ shown in Fig. 1 and its derivatives, bound to small silver clusters $\mathrm{Ag}_{n}$. In doing so we will contrast the conventional DFT-D technique, including D2, D3 corrections, with the hybrid QM:QM approach for obtaining problem-specific $C_{6}$ coefficients for the heavy atoms. Our choice of the system is motivated by the use of $\operatorname{Ir}(\mathrm{III})$ complexes as photosensitizers in photocatalytic water splitting. In Ref. [61], for instance, the homogeneous catalytic system consisting of IrPS combined with the sacrificial reductant triethylamine and a water reduction iron catalyst has been demonstrated. Hybrid systems consisting of IrPS and small metal clusters hold the promise to obtain a heterogeneous catalytic system with improved performance.

The interaction of IrPS with small silver clusters (1-6 silver atoms) and in particular changes in absorption spectra upon binding have been studied in Ref. 62] employing the long-range corrected DFT (LC-BLYP) approach. The obtained results demonstrated strong changes in the absorption spectra of the combined systems as compared with the pure constituents. To proceed with larger metal clusters it is desirable to have a reliable method which properly describes binding interactions at low computational cost such as DFT-D.

The paper is organized as follows: First, we briefly recall the main features of the DFT-D approach and outline the computational details in Section 2. Second, in Section 3 we present the results of fitting $C_{6}$ coefficients for Ir and Ag atoms employing the QM:QM procedure. We proceed with the applications of the new coefficient set to IrPS derivatives. Final conclusions are given in Section 4 . 


\section{Computational Details}

Initial geometry optimizations of $\mathrm{IrPS}-\mathrm{Ag}_{n}, \mathrm{bpy}-\mathrm{Ag}_{n}$, and $\mathrm{ppyH}-\mathrm{Ag}_{n}$ complexes as well as of pure organic and metal parts were carried out using the generalized gradient approximation (GGA) functional of Perdew, Burke, and Ernzerhof (PBE) 63] along with the def2-SV(P) basis set [64, 65]. Optimizations were carried out without any symmetry constraints. Starting geometries of small silver clusters $\mathrm{Ag}_{n}, n=2-20$ were taken from previous studies [66, 67, 68, 69, 70]. On these optimized geometries, single point calculations with PBE, Random Phase Approximation (RPA), and MP2 were done employing the def2-TZV $(\mathrm{P})$ basis set [71, 72].

The binding energy, $E_{\mathrm{b}}$, has been defined as

$$
E_{\mathrm{b}}=E_{\mathrm{tot}}-E_{\mathrm{Ag}_{n}}-E_{\mathrm{IrPS}}
$$

where $E_{\mathrm{tot}}, E_{\mathrm{IrPS}}$, and $E_{\mathrm{Ag}_{n}}$ are the total energies of the relaxed complex, dye molecule, and silver cluster, respectively.

It goes without saying that at the moment for the present system, for example, $\operatorname{CCSD}(\mathrm{T})$ reference calculations are out of reach. Higher order perturbation theory (MP3, MP4) doesn't improve the situation as shown in Ref. [33]. An alternative is the RPA method, which is of slightly higher computational cost as MP2, see [73]. Unlike MP2, the RPA method does not suffer from problems like infinite energies for small bandgap systems and it was shown to provide an adequate description for non-covalent interactions [74]. These authors also pointed out that sufficiently accurate binding energies of weakly bound systems can be obtained only by using complete basis set extrapolation or basis sets larger than quadruple- $\zeta$. But, this level is hardly affordable for heavy elements like Ag or Ir, not to mention that besides regular basis sets one would need auxiliary ones to perform RI-calculations [75, 73]. Therefore, we are forced to restrict our considerations to basis sets of triple- $\zeta$ quality. At least for the case of MP2 we have performed a convergence study and concluded that def2-TZVP binding energies are almost saturated with respect to basis set; e.g. the corresponding binding energy in MP2 of IrPS- $\mathrm{Ag}_{2}$ were $-0.887,-1.000$, and -0.961 eV for def2-SV(P), def2TZVP, and def2-QZVP, respectively.

For the RPA calculations we have employed the resolution-of-the-identity approximation (RI-RPA) for the two-electron integrals [76, 77, 78, 79, 80], the corresponding auxiliary basis sets were taken from Refs. [65, 81, 172, 82]. The RI-RPA calculations of the correlation energy were done non-self-consistently 
on top of the PBE converged set of Kohn-Sham molecular orbitals with the number of grid points set equal to 30. In terms of the notation introduced in [83, the cRPA@PBE approach is applied in this work. For simplicity, we will further use just the abbreviation RPA. In the RPA and MP2 calculations, the frozen-core approximation was used to speed up the calculations and to avoid the using basis sets with additional tight core correlation functions. According to default selection, orbitals below $-3 E_{\text {Hartree }}$ were frozen, i.e. $1 \mathrm{~s}$ of nitrogen and carbon atoms, $4 \mathrm{~s}$ of silver and $5 \mathrm{~s}$ of iridium. All calculations were done with the TURBOMOLE 6.3 program package [84.

We introduced the dispersion term correcting DFT results according to the Grimme DFT-D2 model [85, 28]. Here the total energy of system is given by expression

$$
E_{\mathrm{DFT}-\mathrm{D}}=E_{\mathrm{DFT}}+E_{\mathrm{disp}},
$$

where $E_{\mathrm{DFT}}$ is the energy obtained from the DFT calculation and $E_{\text {disp }}$ is a dispersion term including the $C_{6} R^{-6}$ dependence. $E_{\text {disp }}$ has been determined as introduced by Grimme for DFT-D2 [85, 28]

$$
\begin{gathered}
E_{\text {disp }}=-s_{6} \sum_{i} \sum_{j} \frac{C_{6}^{i j}}{R_{i j}^{6}} f_{\text {damp }}\left(R_{i j}\right) \\
f_{\text {damp }}\left(R_{i j}\right)=\frac{1}{1+\exp \left[-d\left(R_{i j} / R_{r}-1\right)\right]}
\end{gathered}
$$

where $R_{\mathrm{r}}$ is the sum of van der Waals radii of the interacting atoms, $d$ determines the steepness of the damping function, and $C_{6}$ is obtained as

$$
C_{6}^{i j}=\sqrt{C_{6}^{i} C_{6}^{j}} .
$$

The damping function $f_{\text {damp }}$, the scaling factor $s_{6}$, and the atomic $C_{6}^{i}$ coefficients for non-metal atoms have been taken without changes from Ref. 28 .

Atomic coefficients for metal atoms (silver and iridium) have been obtained using the hybrid QM:QM method proposed in Ref. [30] and applied to metal surfaces in Ref. [32]. In this approach, we assume the dispersion energy to be the energy difference between binding energies calculated with the reference (RPA or MP2) and DFT (for discussion see Section 3C). The application of quite large basis sets allows one to neglect the BSSE correction 
as it was pointed out by Grimme [28. Differences in the binding energies between MP2/RPA and PBE for different numbers of silver atoms have been least-square fitted using Eq. (3) in order to obtain atomic coefficients.

Because of the fact that the target systems ( $\left.\operatorname{IrPS}-\mathrm{Ag}_{n}\right)$ include two metal elements the task was divided into two steps. First, we considered structures consisting of the same small silver clusters and phenylpyridin (ppyH) or bipyridin (bpy) molecules. For these cases, we only needed to approximate the dispersion coefficient for silver. This has been done using MP2 and RPA references. Second, we regarded the set of $\mathrm{IrPS}-\mathrm{Ag}_{n}$ structures and did the same fitting procedure applying the coefficient for silver calculated at the first stage. However, for computational reasons this was possible for the MP2 reference only. Alternative to this two step procedure we approximated the two coefficients simultaneously using all dependencies. In this respect, the addition of sets of model structures, including separate ligands as organic part, was reasonable because the interaction between IrPS and silver clusters is mainly due to the dispersion interaction with ligands, with the central Ir atom being shielded.

\section{Results and discussion}

\subsection{Geometries of Weakly Bound Complexes}

For each given combination of silver cluster and organic molecule, we have first optimized from 2 to 5 different structures starting from various initial relative orientations. For brevity, in Fig. 2 only some examples of optimized complexes are plotted, for the full list of structures and their notation see the Supplementary Material [86]. In case of ppyH or bpy aromatic molecules, silver clusters are normally strongly bound to the $\mathrm{N}$ atoms of ppyH and bpy (Fig. 2a), which is impossible when interacting with IrPS where $\mathrm{N}$ atoms are oriented towards the central Iridium atom. Nevertheless, such structures have been also taken into account because we do not want to exclude the possibility of superposition of dispersion interactions. In structures where there is no direct interaction between $\mathrm{Ag}$ and $\mathrm{N}$ atoms, the silver cluster is bound to one of the rings (see Fig. 2b). For the ppyH molecule, in most cases the cluster is attached to the heterocycle. Still another possibility is that one of the silver cluster's planes interacts with the $\pi$ aromatic system and is approximately parallel to the plane of one or both aromatic rings (see Fig. 2ce, d). 
Upon complex formation the bond lengths of the silver clusters, bpy, and $\mathrm{ppyH}$ do not strongly change as compared to gas phase. The most pronounced changes occur in ppyH and bpy torsional angles between the aromatic rings. The changes for bpy are in the range of $1-6^{\circ}$ with several exceptions where these changes achieve values in the range $26-33^{\circ}$. For ppyH, there are even more cases where changes are in the range of $23-35^{\circ}$. This could be due to the fact that the phenyl-ring of ppyH is repelled once the silver cluster comes close to bind with the $\mathrm{N}$ atom. The distances between the closest atoms of the interacting subsystems are 2.3-4.0 $\AA$.

In our previous study on the IrPS bound to small $(n \leq 6)$ silver clusters 62], we found that configurations in which $\mathrm{Ag}_{n}$ is situated in the cavities between ligands are the lowest in energy. Note that in all cases the interactions are "weak" and no chemical bonds are formed. Here we extend this study to $\mathrm{IrPS}-\mathrm{Ag}_{n}$ geometries up to 20 silver atoms, however, focussing on those structures where the cluster is located in the "ppy-ppy" cavity and $n$ is even. Similar to the small systems [62], the geometry optimization was carried out without symmetry constraints, except for some cases where the $\mathrm{C}_{2}$ point symmetry of the IrPS part 87] was retained. Some examples of IrPS $-\mathrm{Ag}_{n}$ complexes can be found in Fig. 3 .

\subsection{Binding Energies}

Binding energies per silver atom of all structures $\left(\mathrm{ppyH}-\mathrm{Ag}_{n}, \mathrm{bpy}-\mathrm{Ag}_{n}\right.$, and IrPS $-\mathrm{Ag}_{n}$ ) selected for further $\mathrm{C}_{6}$ coefficient fitting are plotted in Fig. 4 (details are given in the Supplementary Material [86]). In general the range of binding energies (0.01-0.59 eV) is comparable for all sets of model structures. This corresponds to physisorption of the organic molecule on the silver clusters, with binding energies being about 10 times smaller compared to binding energies of silver atoms within large nanoparticles [88]. From Fig. 4 one can also notice that $E_{\mathrm{b}}$ decreases with increasing number of silver atoms in the system.

Note that the RPA calculations were only computationally affordable for structures containing up to 10 silver atoms and bpy or ppyH but not IrPS as an organic counterpart. Concerning the discrepancies between the PBE and MP2 calculations, it is observed that they are more pronounced for IrPS $-\mathrm{Ag}_{n}$ systems, with MP2 results naturally being always larger than those of PBE. The RPA binding energies for bpy $-\mathrm{Ag}_{n}$ and $\mathrm{ppyH}-\mathrm{Ag}_{n}$ lie in between PBE and MP2 ones, with RPA energies being in average $24 \%$ lower that MP2. 
Next, these data on binding energies are used to optimize the dispersion coefficients.

\subsection{Problem-specific Dispersion Coefficients for Ir and Ag}

Using the QM:QM approach we obtained $C_{6}$ coefficients for $\mathrm{Ag}$ and Ir in two different ways as outlined in the Computational Details section. The coefficients obtained either in two-steps or by simultaneous fitting as well as corresponding root mean squared deviations (RMSD) for binding energy fitting are collected in Table 1. For silver, the dispersion coefficient was obtained using both RPA and MP2 as references. The $C_{6}$ coefficients used in the D2 model [28] are also given in Table 1. It should be noted that for $\mathrm{Ag}$ and Ir only estimates of coefficients are available in D2. For example $C_{6}^{\mathrm{Ag}}$ was assumed to be an average of preceding group VIII and following group III element. The corresponding $C_{6}$ coefficients of the D3 model are also included in the Table 1 but two points should be highlighted before the comparison with the coefficients obtained in the current work. First, they are coordination number dependent; in the table only values which were applied for the systems under investigation are collected. Second, to fit all the dispersion forces in DFT-D3 higher-order terms $C_{8}$ and $C_{10}$ are also used. We also included in Table 1 the values of $C_{6}$ obtained within the combination of dispersion-corrected density-functional theory (the DFT+ van der Waals approach) [35], with the Lifshitz-Zaremba-Kohn theory for the nonlocal Coulomb screening within the bulk [37]. The average value of $C_{6}$ deduced from $\operatorname{CCSD}(\mathrm{T})$ interaction energies between silver dimers at large distances is also provided in Table 1 [89].

Comparing new coefficients based on MP2 reference and standard (D2) coefficients, one notices that the present QM:QM $C_{6}$ coefficients are substantially larger than those of the standard D2 model, with the differences for $\mathrm{Ag}$ being more pronounced than for Ir (increase by $170-180 \%$ vs. $25-70 \%$ ). The two new $C_{6}^{\mathrm{Ag}}$ coefficients are closer to each other than corresponding $C_{6}^{\mathrm{Ir}}$ ones, hinting at the minor influence of Ir coefficient on the approximation of $E_{\mathrm{MP} 2}-E_{\mathrm{PBE}}$ differences for the case of silver.

Here, one should take into account the possible deficiencies of MP2 for predicting binding energies. For example, for benzene molecule the $C_{6}$ coefficient evaluated by MP2 is overestimated by more than $40 \%$ [90]. These overestimations could be even more pronounced for highly polarizable systems such as metal clusters and for periodic systems should lead to infinite $C_{6}$ coefficient because of vanishing bandgap. Indeed, if we compare highly 
accurate $C_{6}$ values from Table 1 of Ref. [37] for the bulk silver within DFTvdWsurf approach which was obtained using the highly accurate experimental dielectric function of the $\mathrm{Ag}$ bulk, our $C_{6}$ is about one magnitude higher. The $C_{6}$ value for free silver atoms from DFT-vdW [35] is about 3 times larger than for the bulk phase [37]. However, all our systems have non-vanishing bandgap even up to $\mathrm{Ag}_{92}$. For test cases (see the Supplementary Material), MP2 overestimates binding energies by only 10-40\% if compared to CCSD. The orbital shift in MP2 leads to almost linear scaling of energy [91] which indirectly evidences the absence of problems with the bandgap. Nevertheless, if one compares the present results with $\operatorname{CCSD}(\mathrm{T})$ for silver dimers, the $C_{6}$ coefficients obtain by MP2 are three times larger. Summarizing, $C_{6}$ coefficients obtained by MP2 are likely to be overestimated, but one can state that for small systems they are larger than for the bulk metal.

Alternative to the MP2 reference we consider RI-RPA/def2-TZVP calculations. As noted before these have been performed for a smaller test set only, which did not include IrPS- $\mathrm{Ag}_{n}$ structures and cases with more than $10 \mathrm{Ag}$ atoms. Separate fitting leads to a substantially different $C_{6}^{\mathrm{Ag}}$ coefficient, which is even smaller (by 28\%) than the corresponding standard D2 value, see Table 1. In order to investigate this point further we performed a test calculation (Test1 in Tab. 1) where the MP2 reference is taken for the same reduced set of structures. This yields a lowering of $C_{6}^{\mathrm{Ag}}$ with respect to the full MP2 set, but still a notably higher value (13\%) as compared with RPA.

To scrutinize the large variability of $C_{6}^{\mathrm{Ir}}$ further two additional fits have been performed. First, we fitted $C_{6}^{\mathrm{Ag}}$ using all sets of test structures (MP2 reference) and assuming $C_{6}^{\mathrm{I} r}=842.0 \mathrm{eV} \AA^{6}$ (D2 model), and second, we applied the standard D2 $C_{6}^{\mathrm{Ag}}=255.69$ and fitted $C_{6}^{\mathrm{Ir} r}$ only for the IrPS- $\mathrm{Ag}_{n}$ set of structures. The results are also shown in Table 1 (entries "Test2", "Test3"). Taking $C_{6}^{\mathrm{I} r}=842.0 \mathrm{eV} \AA^{6}$ leads to a slight increase of $C_{6}^{\mathrm{A} g}$ if compared to the "simultaneous" value, with the quality of fitting being practically the same. Setting $C_{6}^{\mathrm{Ag}}=255.69$ led to an enormously increased $C_{6}^{\mathrm{I} r}$, with the RMSD increasing dramatically as well. This finding can be rationalized as follows: The Ir atom is situated in the center of photosensitizer and hence screened by the ligands what hinders a direct interaction with the silver clusters. To cover the same amount of dispersion with the standard fixed value of $C_{6}^{\mathrm{Ag}}$ is only possible with an extremely large coefficient for iridium. Hence, it can be argued that the actual coefficients are extremely sensitive to the chemical environment, what in principle makes the simultaneous fit 
more reliable. The dependency of coefficients on environment hints that in the current approach they are more akin to those of DFT $+\mathrm{vdW}, \mathrm{XDM}$ and more recent methods like DFT $+\mathrm{MBD}$, DFT+vdWsurf (for discussion see [92]) where screening effects are considered in a more complicated way.

Fitting the difference between PBE and a reference method (MP2 or RPA) invokes naturally the question, which effects and contributions are actually included in the resulting dispersion energy. DFT-D2 describes solely dipole-dipole interactions, whereas DFT-D3 additionally takes triple-dipole interactions into account. MP2 provides an approximate description of all vdW effects with moderate computational costs but it is known to overestimate dispersion effects. In Ref. [90], a dispersion-corrected MP2 version was suggested to overcome this deficiency. Finally, as a consequence of its nonlocality the RPA approach should describe the long-range vdW-interaction in the most accurate way. Inclusion of dynamic electronic screening extends the applicability of RPA to small-gap and metallic systems, which cannot be treated generally by MP2.

According to the original Grimme DFT-D2 model, the dispersion contribution is additionally multiplied by a damping function and a global scaling factor $s_{6}$ which is functional-dependent. In that way, the strength of dispersion interaction is adjusted for different $\mathrm{XC}$ functionals. In case of PBE, $s_{6}=0.75$ is used. Such an ad hoc treatment is justified at short distances where it provides a correction to the overestimated dispersion forces, but it has no direct meaning at long distances. The Fermi-type damping function includes an additional empirical parameter $d$, the steepness of the damping function which is universal for all functionals. As it was already pointed out in Sec. 1, the damping function is needed to smoothly switch-off the dispersion term at short distances. The choice of a particular type of damping function has no crucial impact on the results (for a discussion see Ref. 34]). In more advanced DFT-D variants such as DFT-D3, TS, and XDM, there is no direct scaling of $C_{6}$ coefficients, but damping functions include more empirical parameters, like (order-dependent-) coefficients which scale vdW (or cutoff) radii for each density functional applied. Furthermore, the cutoff radius itself is also an arbitrary parameter that is not universal for different DFT-D approaches and should be chosen separately. In DFT-D3, the $s_{8}$ parameter is used to scale the contribution of triple-dipole interactions and $s_{6}$-scaling is applied for double-hybrid density functionals. Summarizing, all DFT-D schemes include empirical corrections justified by physical meaning and by fitting to experimental or more precise computational results. In the 


\begin{tabular}{ccc}
\hline & Ag & Ir \\
\hline D2 [28] & 255.69 & 842.00 \\
D3 [34] & $160.53(\mathrm{CN}=1)$ & $182.60(\mathrm{CN}=3)$ \\
Ruiz [37] & $72.91(\text { free } 202.60)^{*}$ & - \\
Hatz [89] & 216.96 & - \\
Separate (MP2) & $690.41(0.08)$ & $1420.60(0.11)$ \\
Simultaneous (MP2) & $717.70(0.09)$ & $1051.68(0.09)$ \\
Separate (RPA) & $183.18(0.06)$ & - \\
Test1 & $599.46(0.05)$ & - \\
Test2 & $727.92(0.09)$ & 842.00 \\
Test3 & 255.69 & $45667.49(0.24)$ \\
\hline
\end{tabular}

Table 1: Values of $C_{6}^{\mathrm{Ag}}$ and $C_{6}^{\mathrm{Ir}}\left(\right.$ in $\mathrm{eV} \AA^{6}$ ) fitted separately and simultaneously with the QM:QM method (RMSD of $E_{\mathrm{r} e f}-E_{\mathrm{PBE}-\mathrm{D} *}$ in $\mathrm{eV}^{2}$ are given in parenthesis) and from literature. For D3 value, the coefficients are only given for coordination numbers $(\mathrm{CN})$ in parenthesis. *The unscreened (free) value from [35] is given for comparison. For the meaning of Tests see text.

present treatment we do not aim at refitting the scaling factor and take the DFT-D2 model as developed previously.

In the following applications we continue to consider two sets of coefficients: First the MP2 result obtained from simultaneous fitting (PBE-D2*). This includes the complete set of test structures which is not available for the most likely more accurate RPA. Note, however, since no exact reference is available the judgement concerning accuracy of the two methods is solely based on results reported for other systems in literature. Therefore MP2 derived results will be contrasted to RPA ones which are supplemented by the $C_{6}^{\mathrm{Ir}}$ coefficient taken from the D2 model (PBE-D2**).

\subsection{Applications}

First, the obtained coefficients for $\mathrm{Ag}$ and Ir were verified for dissociation of two complexes: $\mathrm{IrPS}-\mathrm{Ag}_{4}$ and $\mathrm{IrPS}-\mathrm{Ag}_{20}$. The optimized (PBE/def2$\mathrm{SV}(\mathrm{P})$ ) geometries were frozen and distances between Ir and closest Ag atoms were changed. Single point calculations of obtained structures were done using PBE (pure and in different dispersion-included variants) and MP2 with the def2-SV $(\mathrm{P})$ basis set.

The dependencies of calculated binding energies on the Ir-Ag distance are plotted in Fig. 5. Naturally, a very good agreement between MP2 and 
PBE-D2* curves can be concluded for both complexes, with the D2, D3, and $\mathrm{D} 2{ }^{* *}$ lying in the middle of MP2-PBE gap. Note that the minima of MP2 and DFT-D2* curves do not coincide since a larger basis set has been used for obtaining the $\mathrm{C}_{6}$ coefficients. RPA based results as well as coefficients themselves are lying between corresponding PBE-D2 and PBE-D3 curves, demonstrating slightly slower binding energies closer to dissociation limit.

As a second application, we evaluated the binding energy of the large nearly spherical silver cluster $\mathrm{Ag}_{92}$. The initial geometry of this cluster was cut from the fcc bulk silver and optimized with PBE/def2-SV(P) separately. Then the silver cluster was placed in the ppy-ppy cavity of the photosensitizer analogous to the smaller systems. The constructed complex was partially optimized using PBE-D2*, i.e. only those silver atoms nearest to the IrPS and all atoms of IrPS were allowed to relax upon optimization. (Note for the purposes of comparison, we also performed an optimization with PBE-D2 and PBE-D3; the changes in geometries are only minor (up to $0.06 \AA$ and $0.2^{\circ}$ ) and can be neglected.) The resulting geometry is shown in Fig. 6 together with the corresponding binding energies per one silver atom calculated within different PBE-D models. The IrPS- $\mathrm{Ag}_{92}$ complex has no symmetry and the distance between central Ir atom and nearest silver atom is $5.2 \AA$ which is slightly higher than in the case of small silver clusters. Due to steric reasons the large silver cluster can not come closer without significant distortions in its shape. The binding energy of the largest investigated system per one silver atom is very small and in the region of $0.004-0.021 \mathrm{eV}$ for all PBE$\mathrm{D}$ variants. Similar to the small systems, the account for dispersion forces increases the binding energy $E_{\mathrm{b}}$ : from 0.004 (PBE) to 0.012 (both PBE-D2 and PBE-D3, PBE-D2**) and to $0.021 \mathrm{eV}$ (for PBE-D2*). The obtained value also confirms the decrease of $E_{\mathrm{b}}$ per 1 silver atom with increasing system size. The trend can be clearly seen in Fig. 6 where binding energies for selected examples of IrPS- $\mathrm{Ag}_{\mathrm{n}}$ calculated with MP2 and all the PBE-D variants (within def2-TZVP basis set) are plotted. For clarity, only cases with the largest binding energies within MP2 are selected.

Finally, we apply the new coefficients to derivatives of IrPS. Although our coefficients are by construction suited for the particular photosensitizer with ppy and bpy ligands (isolated or located around Ir central atom) we wanted to scrutinise the sensitivity with respect to the type of heteroatom. For this reason we investigated the binding between and $\mathrm{Ag}_{10}$ cluster an a photosensitizers containing oxygen $\left(\left[\operatorname{Ir}(\mathrm{op})_{2}(\mathrm{bpy})\right]^{+}\right)$and sulphur $\left(\left[\operatorname{Ir}(\mathrm{bt})_{2}(\mathrm{bpy})\right]^{+}\right)$in the ligands. The structures of optimized complexes are depicted in Fig. 7 and 
they are overall similar to those of IrPS. Again the silver cluster is located in the cavities between the two ligands, with the distance between the central Ir atom and the nearest silver atom being about 4.8-4.9 $\AA$. The binding energies are about $1.4 \mathrm{eV}$ (or $0.14 \mathrm{eV}$ per one silver atom) for both cases, which is only slightly lower than those of $\operatorname{IrPS}-\mathrm{Ag}_{10}$ and corresponds again to a weak interaction (physisorption).

\section{Conclusions}

The interaction of $\operatorname{Ir}(\mathrm{III})$ photosensitizers containing ppy and bpy ligands with small silver clusters $\mathrm{Ag}_{n}(n=2-20)$ is studied using dispersion-corrected density functional theory together with the RPA and MP2 methods. The goal has been to develop a system-specific set of $C_{6}$ parameters for Ir and $\mathrm{Ag}$ atoms in the spirit of the D2 correction. To this end the QM:QM scheme was employed for a set of model structures. An important aspect for this particular type of system is that the Ir atom is shielded by the ligands. As a consequence the Ir parameters turn out to be rather sensitive to the actual method of fitting, whereas the $\mathrm{Ag}$ coefficients are more robust.

In general binding of silver clusters to IrPS is weak and in the physisorption range. The binding energy per silver atom decreases with the size of the cluster to become as small as $0.01-0.02 \mathrm{eV}$ for the largest cluster studied $(n=92)$.

Although our new coefficients are by construction suited for the particular example of interaction of silver clusters with organic or metalorganic molecules containing ppy and bpy ligands, the transferability of new $C_{6}$ coefficients for similar systems was shown using the exemplary cases of IrPS containing $\mathrm{O}$ and $\mathrm{S}$ heteroatoms.

In order to derive $C_{6}$ two references have been considered, i.e. MP2 and RPA. It was found that MP2 did not give convergence and bandgap-related problems, most likely since the considered metal clusters still have molecular character and can be described with respective orbitals. However, based on reports in literature one would expect that RPA is more reliable for the description of binding in these weakly bound organic/inorganic hybrid systems. Therefore, the $C_{6}^{\mathrm{Ag}}$ value obtained on the RPA level should be considered as being more accurate compared to MP2. This value turns out to be close to the ones of the Grimme D2/D3 sets. However, this similarity should be taken with care. In view of the fact that the polarizability is determined by a non-local response kernel, the reduction to a single coefficient for an arbitrary 
arrangement of atoms is quite an approximation. As an additional caveat we should mention that, of course, extraction of dispersion coefficients in the present QM:QM scheme assumes that other contributions to the interaction do not differ between the QM methods. This is not necessarily the case and differences might result from electrostatic or induction contributions. Therefore, in view of the size of the systems considered here, crucial tests against experimental data will be required for further validation.

\section{Acknowledgements}

This work has been financially supported by the ESF project "Nanostructured Materials for Hydrogen Production (Nano4-Hydrogen)" and the BMBF project "Light2Hydrogen" ("Spitzenforschung und Innovation in den Neuen Ländern").

\section{References}

[1] E. Dulkeith, A. Morteani, T. Niedereichholz, T. Klar, J. Feldmann, S. Levi, F. van Veggel, D. Reinhoudt, M. Möller, D. Gittins, Phys. Rev. Lett. 89 (2002) 203002.

[2] P. Anger, P. Bharadwaj, L. Novotny, Phys. Rev. Lett. 96 (2006) 113002.

[3] V. N. Pustovit, T. V. Shahbazyan, Phys. Rev. B 83 (2011) 085427.

[4] G. Zweigle, J. McHale, J. Phys. Chem. C 115 (2011) 13693-13703.

[5] A. Bilić, J. R. Reimers, N. S. Hush, J. Phys. Chem. B 106 (2002) 6740.

[6] A. Bilić, J. R. Reimers, N. S. Hush, R. C. Hoft, M. J. Ford, J. Chem. Theor. Comp. 2 (2006) 1093.

[7] S. Higai, J. Nara, T. Ohno, Surf. Sci. 600 (2006) 685.

[8] S. Kristyán, P. Pulay, Chem. Phys. Let. 229 (1994) 175-180.

[9] P. Hobza, J. Šponer, T. Reschel, J. Comp. Chem. 16 (1995) 1315-1325.

[10] J. Pérez-Jordá, A. Becke, Chem. Phys. Let. 233 (1995) 134-137.

[11] J. Pérez-Jordá, E. San-Fabián, A. Pérez-Jiménez, J. Chem. Phys. 110 (1999) 1916-1920. 
[12] S. Grimme, Comp. Mol. Sci. 1 (2011) 211.

[13] A. Tkatchenko, L. Romaner, O. Hofmann, E. Zojer, C. Ambrosch-Draxl, M. Scheffler, MRS Bull. 35 (2010) 435-442.

[14] J. Klimeš, A. Michaelides, J. Chem. Phys. 137 (2012) 120901.

[15] O. A. von Lilienfeld, I. Tavernelli, U. Rothlisberger, D. Sebastiani, Phys. Rev. Lett. 93 (2004) 153004.

[16] O. von Lilienfeld, I. Tavernelli, U. Rothlisberger, D. Sebastiani, Phys. Rev. B 71 (2005) 195119.

[17] Y. Y. Sun, Y.-H. Kim, K. Lee, S. B. Zhang, J. Chem. Phys. 129 (2008) 154102.

[18] E. R. Johnson, I. D. Mackie, G. A. DiLabio, J. Phys. Org. Chem. 22 (2009) 1127-1135.

[19] O. von Lilienfeld, Mol. Phys. 111 (2013) 2147-2153.

[20] A. Becke, E. Johnson, J. Chem. Phys. 122 (2005) 154104.

[21] A. Becke, E. Johnson, J. Chem. Phys. 124 (2006) 014104.

[22] J. Ángyán, J. Phys. Chem. 127 (2007) 024108.

[23] A. Becke, E. Johnson, J. Chem. Phys. 127 (2007) 154108.

[24] A. Heßelmann, J. Chem. Phys. 130 (2009) 084104.

[25] S. Steinmann, C. Corminboeuf, J. Chem. Theor. Comp. 6 (2010) 1990.

[26] A. Heßelmann, J. Chem. Phys. 136 (2012) 014104.

[27] H. B. G. Casimir, D. Polder, Phys. Rev. 73 (1948) 360-372.

[28] S. Grimme, J. Comp. Chem. 27 (2006) 1787-1799.

[29] R. Eisenschitz, F. London, Z. Physik 60 (1930) 491.

[30] C. Tuma, J. Sauer, Chem. Phys. Let. 387 (2004) 388.

[31] C. Tuma, J. Sauer, Phys. Chem. Chem. Phys. 8 (2006) 3955. 
[32] Q.-M. Hu, K. Reuter, M. Scheffler, Phys. Rev. Lett. 98 (2007) 176103.

[33] K. Tonigold, A. Groß, J. Chem. Phys. 132 (2010) 224701.

[34] S. Grimme, J. Chem. Phys. 132 (2010) 154104.

[35] A. Tkatchenko, M. Scheffler, Phys. Rev. Lett. 102 (2009) 073005.

[36] A. Tkatchenko, R. DiStasio, R. Car, M. Scheffler, Phys. Rev. Lett. 108 (2012) 236402.

[37] V. Ruiz, W. Liu, E. Zojer, M. Scheffler, A. Tkatchenko, Phys. Rev. Lett. 108 (2012) 146103.

[38] M. Dion, H. Rydberg, E. Schroder, D. Langreth, B. Lundqvist, Phys. Rev. Lett. 92 (2004) 246401.

[39] D. Langreth, M. Dion, H. Rydberg, E. Schröder, P. Hyldgaard, B. Lundqvist, Int. J. Quant. Chem. 101 (2005) 599.

[40] K. Lee, E. D. Murray, L. Kong, B. I. Lundqvist, D. C. Langreth, Phys. Rev. B 82.

[41] O. Vydrov, T. Voorhis, Phys. Rev. Lett. 103 (2009) 063004.

[42] O. Vydrov, T. Voorhis, J. Chem. Phys. 132 (2010) 164113.

[43] T. Sato, T. Tsuneda, K. Hirao, J. Chem. Phys. 126 (2007) 234114.

[44] G. Román-Pérez, J. M. Soler, Phys. Rev. Lett. 103 (2009) 096102.

[45] A. Gulans, M. Puska, R. Nieminen, Phys. Rev. B 79 (2009) 201105.

[46] W. Liu, J. Carrasco, B. Santra, A. Michaelides, M. Scheffler, A. Tkatchenko, Phys. Rev. B 86 (2012) 245405.

[47] O. A. Vydrov, T. Van Voorhis, Phys. Rev. A 81 (2010) 062708.

[48] L. Burns, A. Vázquez-Mayagoitia, B. Sumpter, C. Sherrill, J. Chem. Phys. 134 (2011) 084107.

[49] N. Marom, A. Tkatchenko, M. Rossi, V. V. Gobre, O. Hod, M. Scheffler, L. Kronik, J. Chem. Theor. Comp. 7 (2011) 3944. 
[50] M. Steinmetz, S. Grimme, Chem. Open 2 (2013) 115-124.

[51] L. Goerigk, J. Chem. Theor. Comp. (2014) DOI: 10.1021/ct500026v.

[52] N. Atodiresei, V. Caciuc, P. Lazić, S. Blügel, Phys. Rev. Lett. 102 (2009) 136809.

[53] N. Atodiresei, V. Caciuc, J.-H. Franke, S. S. Blügel, Phys. Rev. B 78 (2008) 045411.

[54] E. McNellis, J. Meyer, R. Reuter, Phys. Rev. B 80 (2009) 205414.

[55] M.-T. Nguyen, C. Pignedoli, M. Treier, R. Fasel, D. Passerone, Phys. Chem. Chem. Phys. 12 (2010) 992.

[56] R. Gaspari, C. Pignedoli, R. Fasel, M. Treier, D. Passerone, Phys. Rev. B 82 (2010) 041408.

[57] E. McNellis, C. Bronner, J. Meyer, M. Weinelt, P. Tegeder, K. Reuter, Phys. Chem. Chem. Phys. 12 (2010) 6404.

[58] A. Antony, C. Hakanoglu, A. Asthagiri, J. Weaver, J. Chem. Phys. 136 (2012) 054702.

[59] R. Kang, H. Chen, S. Shaik, J. Yao, J. Chem. Theor. Comp. 7 (2011) 4002.

[60] S. Ehrlich, J. Moellmann, W. Reckien, T. Bredow, S. Grimme, ChemPhysChem 12 (2011) 3414.

[61] F. Gärtner, B. Sundararaju, A.-E. Surkus, A. Boddien, B. Loges, H. Junge, P. H. Dixneuf, M. Beller, Angew. Chem. Int. Ed. 48 (2009) 9962.

[62] O. Bokareva, S. Bokarev, O. Kühn, Phys. Chem. Chem. Phys. 14 (2012) 4977.

[63] J. P. Perdew, K. Burke, M. Ernzerhof, Phys. Rev. Lett. 77 (1996) 3965.

[64] A. Schäfer, H. Horn, R. Ahlrichs, J. Chem. Phys. 97 (1992) 2571.

[65] K. Eichkorn, F. Weigend, O. Treutler, R. Ahlrichs, Theor. Chim. Acta 97 (1997) 119. 
[66] V. Bonačić-Koutecký, L. Češpiva, P. Fantucci, J. Koutecký, J. Chem. Phys. 98 (1993) 7981-7994.

[67] V. Bonačić-Koutecky, V. Veyret, R. Mitrić, J. Chem. Phys. 115 (2001) 10450-10460.

[68] R. Fournier, J. Chem. Phys. 115 (2001) 2165-2177.

[69] M. Harb, F. Rabilloud, D. Simon, A. Rydlo, S. Lecoultre, F. Conus, V. Rodrigues, C. Félix, J. Chem. Phys. 129 (2008) 194108.

[70] K. Baishya, J. Idrobo, S. Ö̆üut, M. Yang, K. Jackson, J. Jellinek, Phys. Rev. B 78 (2008) 075439.

[71] F. Weigend, R. Ahlrichs, Phys. Chem. Chem. Phys. 7 (2005) 3297-3305.

[72] F. Weigend, M. Häser, H. Patzelt, R. Ahlrichs, Chem. Phys. Let. 294 (1998) 143-152.

[73] H. Eshuis, J. E. Bates, F. Furche, Theor. Chim. Acta 131 (2012) 1084.

[74] H. Eshuis, F. Furche, J. Chem. Phys. 136 (2012) 084105.

[75] H. Eshuis, J. Yarkony, F. Furche, J. Chem. Phys. 132 (2010) 234114.

[76] F. Weigend, Phys. Chem. Chem. Phys. 4 (2002) 4285-4291.

[77] M. Sierka, A. Hogekamp, R. Ahlrichs, J. Chem. Phys. 118 (2003) 9136.

[78] F. Weigend, M. Häser, Theor. Chim. Acta 97 (1997) 331-340.

[79] C. Hättig, F. Weigend, J. Chem. Phys. 113 (2000) 5154.

[80] C. Hättig, A. Hellweg, A. Köhn, Phys. Chem. Chem. Phys. 8 (2006) 1159.

[81] K. Eichkorn, O. Treutler, H. Öhm, M. Häser, R. Ahlrichs, Chem. Phys. Let. 242 (1995) 652.

[82] A. Hellweg, C. Hättig, S. Höfener, W. Klopper, Theor. Chim. Acta 117 (2007) 587.

[83] X. Ren, A. Tkatchenko, P. Rinke, M. Scheffler, Phys. Rev. Lett. 106 (2011) 153003. 
[84] TURBOMOLE V6.3 2011, a development of University of Karlsruhe and Forschungszentrum Karlsruhe GmbH, 1989-2007, TURBOMOLE $\mathrm{GmbH}$, since 2007; available from http://www . turbomole.com.

[85] S. Grimme, J. Comp. Chem. 25 (2004) 1463.

[86] See Supplementary Material Document No. for binding energies of all investigated structures (Table S1), the full list of optimized geometries and corresponding names for bpy $-\mathrm{Ag}_{n}$ (Fig. S1), ppyH- $\mathrm{Ag}_{n}$ (Fig. S2), and $\mathrm{IrPS}-\mathrm{Ag}_{n}$ (Fig.S3).

[87] K. King, R. Watts, J. Amer. Chem. Soc. 109 (1987) 1589.

[88] E. Fernández, J. Soler, I. Garzón, L. Balbás, Phys. Rev. B 70 (2004) 165403.

[89] R. Hatz, M. Korpinen, V. Hänninen, L. Halonen, J. Phys. Chem. A 116 (2012) 11685-11693.

[90] A. Tkatchenko, R. A. DiStasio, M. Head-Gordon, M. Scheffler, J. Chem. Phys. 131 (2009) 094106.

[91] B. O. Roos, K. Andersson, Chem. Phys. Let. 245 (1995) 215-223.

[92] T. Bučko, S. Lebègue, J. Hafner, J. G. Ángyán, Phys. Rev. B 87 (2013) 064110. 


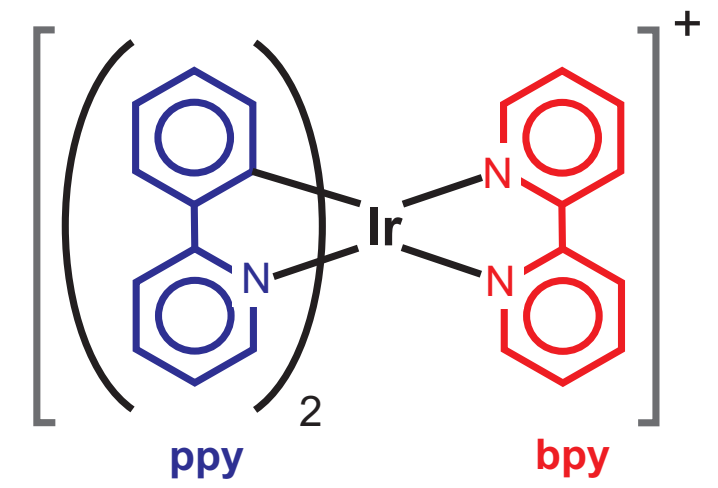

Figure 1: Chemical formula of $\left[\operatorname{Ir}(\text { ppy })_{2}(\text { bpy })\right]^{+}$. 

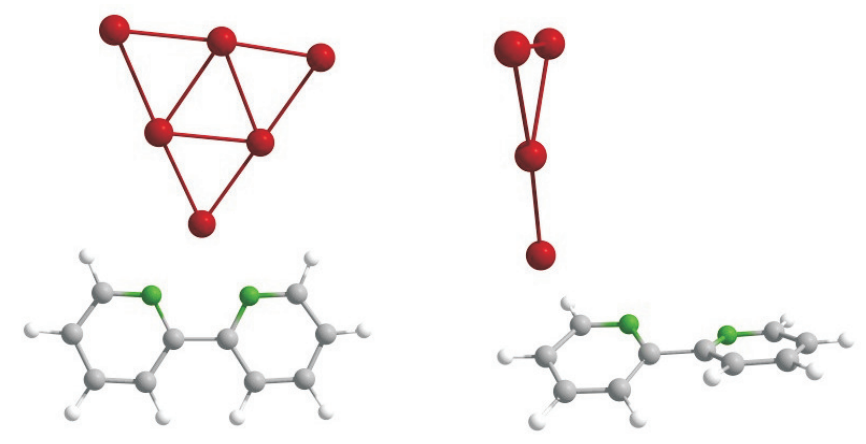

a bpy-Ag6a-1
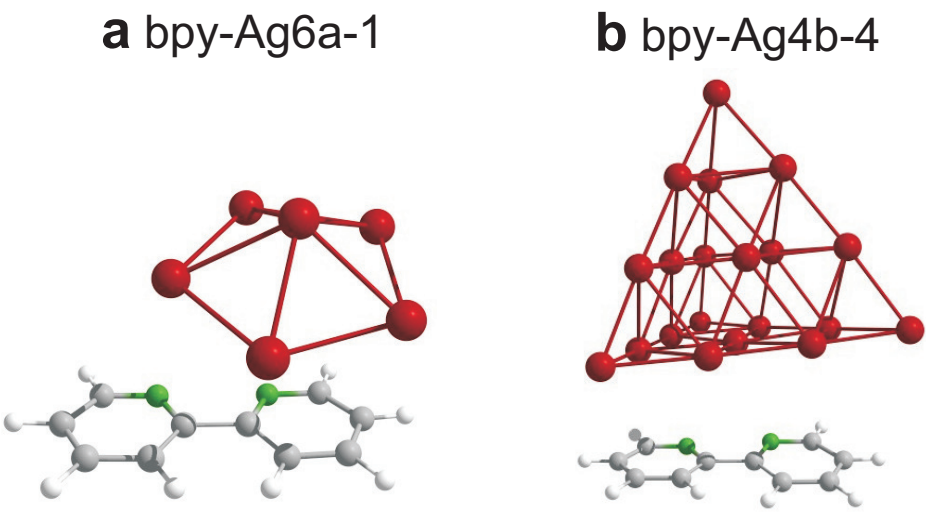

c bpy-Ag6b-3

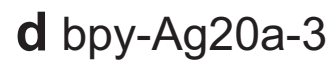

Figure 2: Some representative examples of $\mathrm{bpy}-\mathrm{Ag}_{n}$ optimized geometries (for a full list including the nomenclature see Supplementary Material). 

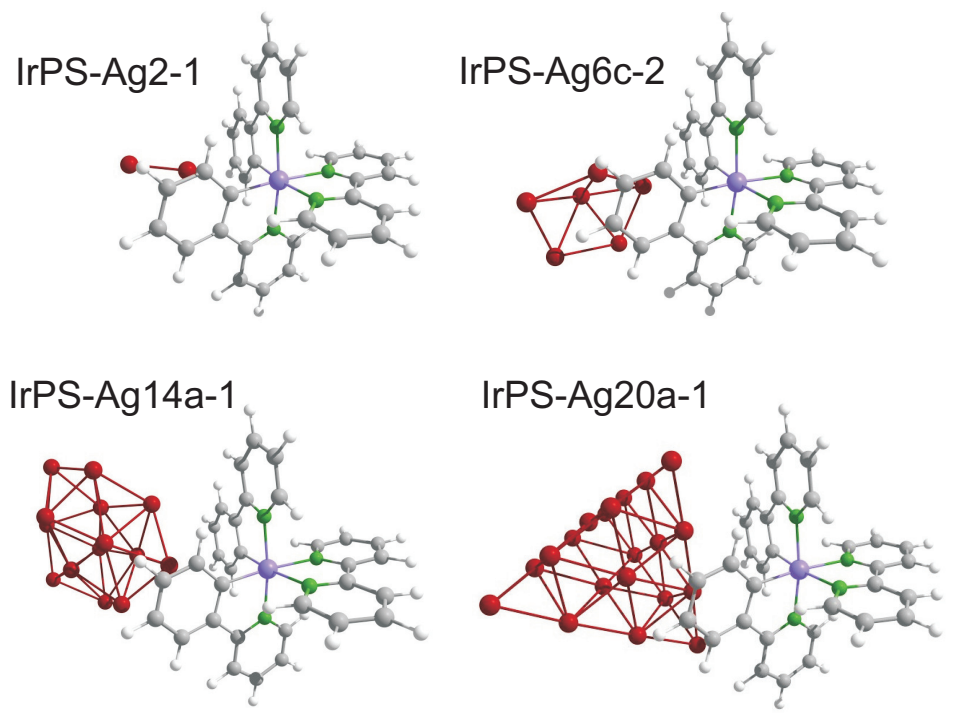

Figure 3: Some examples of optimized IrPS- $\mathrm{Ag}_{n}$ structures (for a full list see Supplementary Material [86]). 

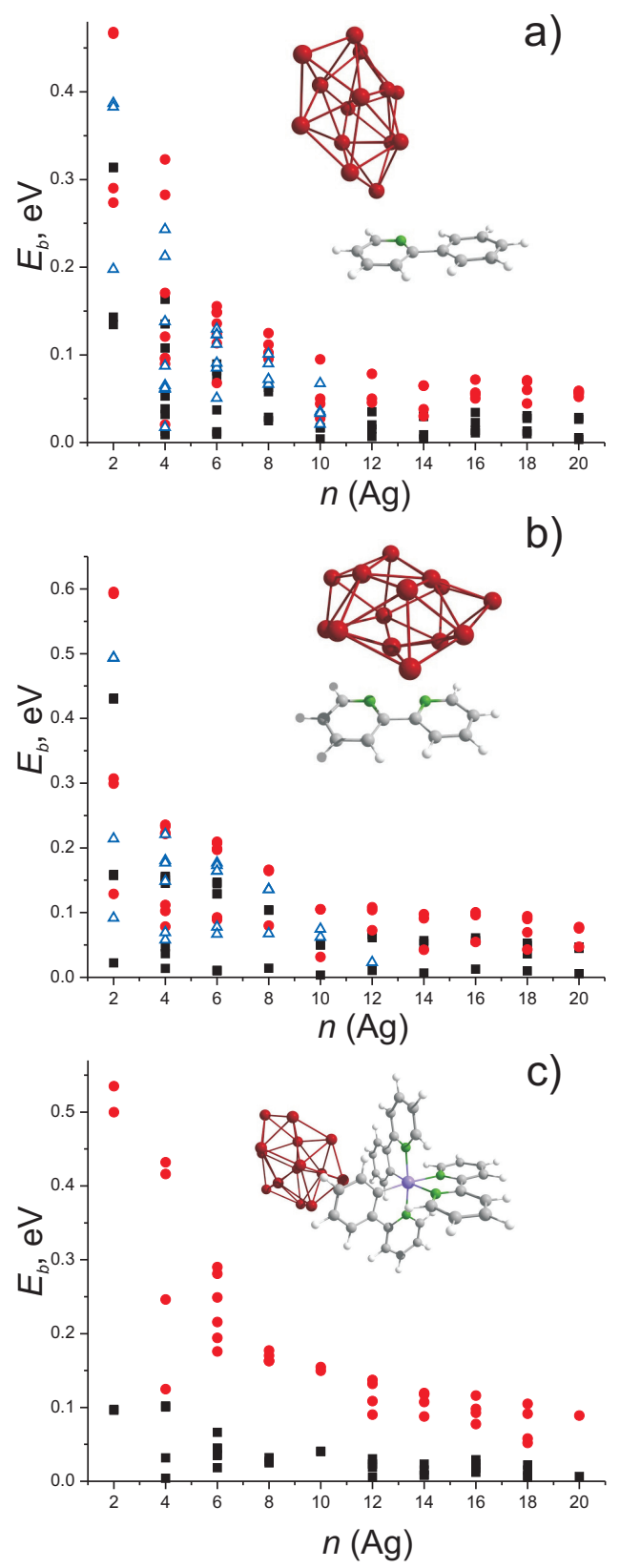

Figure 4: Binding energies per silver atom of all model structures under study: a) ppyH $-\mathrm{Ag}_{n}$, b) bpy $-\mathrm{Ag}_{n}$, c) IrPS $-\mathrm{Ag}_{n}$. Black squares: PBE, blue hollow triangles: RPA, red circles: MP2. For illustration some examples for $\mathrm{Ag}_{14}$ are shown. Notice that for simplicity, we do not mark the corresponding points (PBE, RPA, MP2) for each structure. 

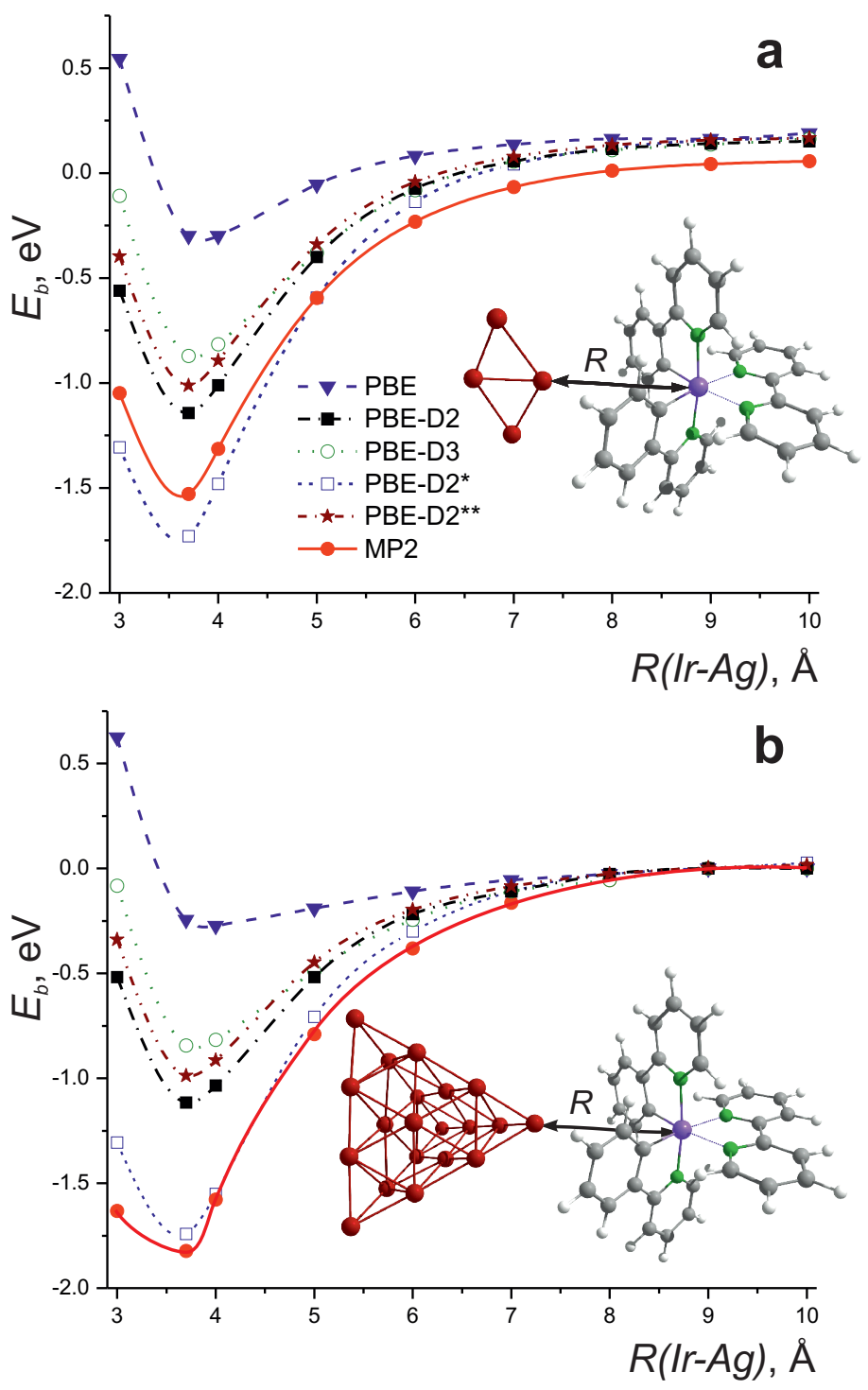

Figure 5: Dissociation curves of $\operatorname{IrPS}-\mathrm{Ag}_{4}$ (a) and $\operatorname{IrPS}-\mathrm{Ag}_{20}$ (b) complexes calculated with MP2 and different PBE variants using the def2-SV(P) basis set. The legend for (b) part is the same as for (a); connecting lines are just a guide to the eyes. 


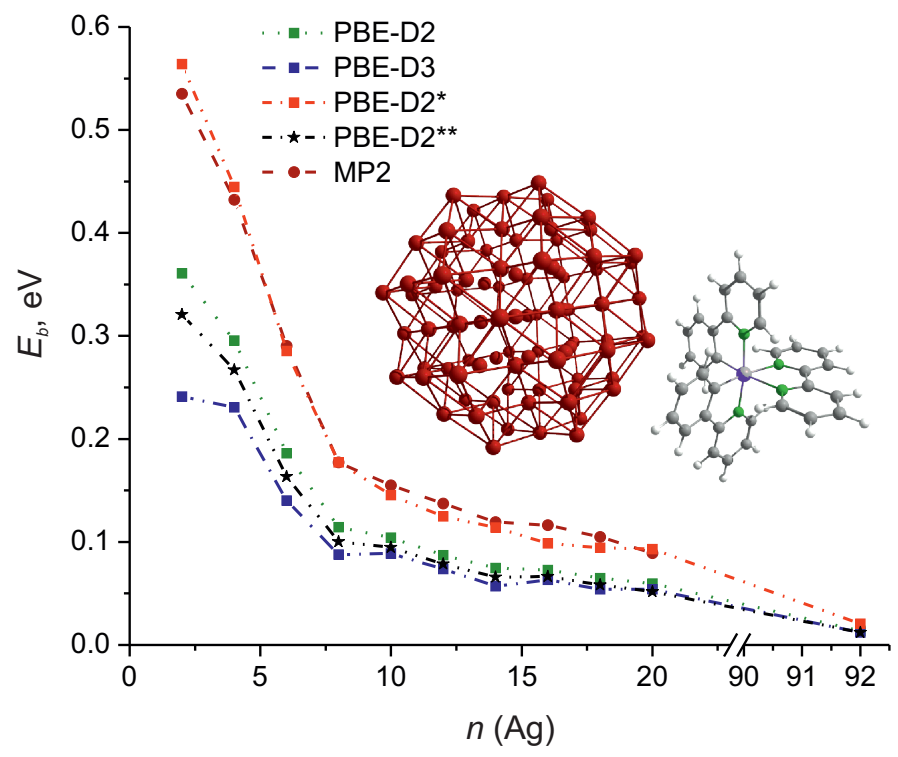

Figure 6: Geometry of optimized complex $\mathrm{IrPS}-\mathrm{Ag}_{92}$ as well as its binding energies evaluated with PBE-D approaches compared to the MP2 binding energies (def2-TZVP basis set). For comparison binding energies for smaller systems are given as well. 

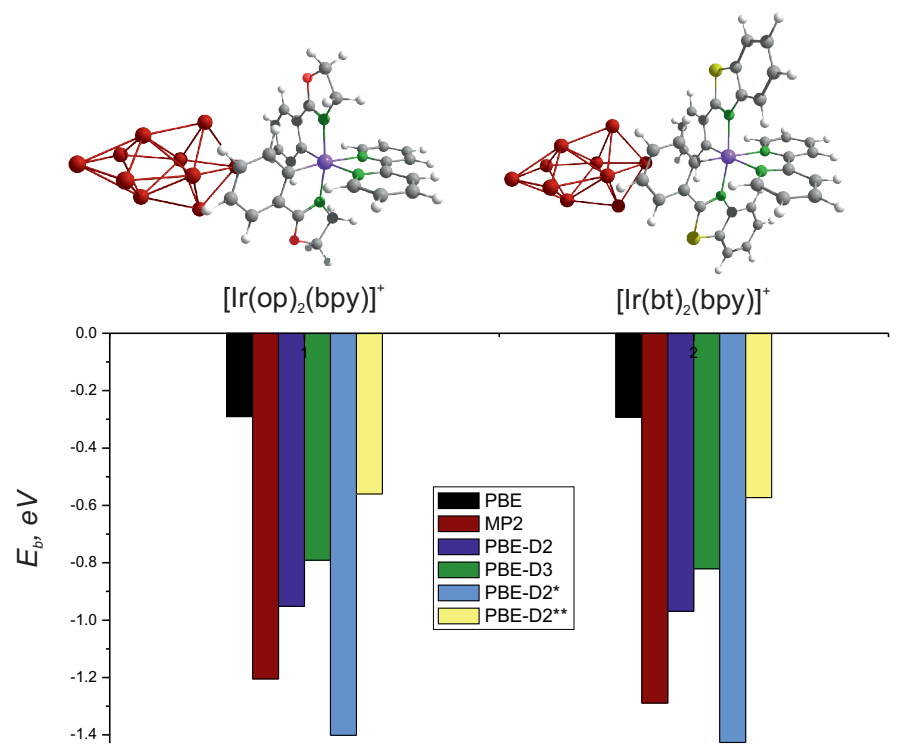

Figure 7: Geometries and comparison of binding energies of two further photosensitizers containing $\mathrm{O}$ or $\mathrm{S}$ atoms using the def2-SV(P) basis set. 